\title{
Effect of Spin-1 Impurities in Dimerized Heisenberg Chains
}

\author{
P. M. Hansen ${ }^{1}$, J. A. Riera ${ }^{1}$, A. Delia ${ }^{2}$, and E. Dagotto ${ }^{2}$ \\ ${ }^{1}$ Instituto de Física Rosario (CONICET) and Departamento de Física, \\ Avenida Pellegrini 250, 2000 Rosario, Argentina \\ ${ }^{2}$ National High Magnetic Field Lab and Department of Physics, Florida State University, \\ Tallahassee, Florida 32306, USA
}

\begin{abstract}
The effects of spin-1 impurities introduced in dimerized antiferromagnetic spin chains are studied using Lanczos diagonalization and quantum Monte Carlo simulations. Firstly, the magnitude of the interaction between a Ni-ion replacing a $\mathrm{Cu}$-ion is derived for the case study system $\mathrm{Cu}-\mathrm{O}-\mathrm{Cu}$. Then, it is shown that the introduction of $S=1$ impurities leads to the suppression of the original spin gap of the dimerized system and to the enhancement of the antiferromagnetic correlations close to the impurity. These features are interpreted in terms of a valence bond picture of the ground state of both the pure and the doped system. The similitudes and differences between the underlying mechanisms that produce these effects in the cases of nonmagnetic and $S=1$ impurities are analyzed. It is stressed that a singlet formed between the spin-1 impurity site and the two spin$1 / 2$ neighbors spins behaves as an effective nonmagnetic vacancy which may be partially responsible for the enhancement of the antiferromagnetic correlations on the whole system. Implications of these results for experiment are also discussed.

PACS numbers: 75.10.Jm, 75.50.Ee, 75.30.Hx, 75.40.Mg
\end{abstract}

\section{INTRODUCTION}

One-dimensional or quasi-one-dimensional magnetic systems have several fascinating properties which continue to attract an intense theoretical activity. One of these properties is the presence of a spin gap in antiferromagnetic Heisenberg chains with integer spin [1]:2] and with spin-1/2 in ladder geometries [3, 4], as well as in chains with frustrating interactions [5], and in the presence of dimerization. In the latter systems, the dimerization frequently appears as a consequence of the spinphonon coupling, i.e. it is due to a spin-Peierls (SP) instability. The interest in the spin-Peierls phenomena was recently revived after the first inorganic SP compound, $\mathrm{CuGeO}_{3}$, was found [6]. A considerable theoretical effort has been devoted to understanding the properties of this compound [7,8]. Most of these studies have been based on a one-dimensional (1D) dimerized Heisenberg model, i.e. retaining only the spin degrees of freedom and neglecting the spin-phonon dynamics. Although most dimerized systems are spin-Peierls systems, there are also several compounds where the intrinsic structure leads to dimerized spin chains. Actually $\mathrm{Cu}\left(\mathrm{NO}_{3}\right)_{2} \cdot 2.5 \mathrm{H}_{2} \mathrm{O}$ was the first inorganic alternating-chain system to be investigated [9]. More recently, inelastic neutron scattering measurements and susceptibility data indicate that the compound $\mathrm{CuWO}_{4}$ consists of weakly coupled alternating chains 10. Another example of structural dimerization is provided by the $\mathrm{CuO}_{2}$ chains which are present in $\mathrm{Sr}_{14} \mathrm{Cu}_{24} \mathrm{O}_{41}$ 11 which also contains $\mathrm{Cu}_{2} \mathrm{O}_{3}$ ladders. In addition, the compound $(\mathrm{VO})_{2} \mathrm{P}_{2} \mathrm{O}_{7}$, which was previously believed to be composed by two-leg spin ladders, is actually better described by alternating spin chains [12].

The experimental introduction of impurities in these magnetic systems has provided additional information in the quest to examine the nature of their ground state and excitations 13. In particular, inorganic systems, such as $\mathrm{CuGeO}_{3}$, can be easily doped with both nonmagnetic and magnetic impurities i.e. by partially replacing the $\mathrm{Cu}^{2+}$ ions by $\mathrm{Zn}^{2+}$ and $\mathrm{Ni}^{2+}$ ions, respectively. The effect of impurities on $\mathrm{CuGeO}_{3}$ has been investigated using neutron scattering [14, 15], magnetic susceptibility measurements [14,16], specific heat measurements [17, and spin electron resonance [18]. The main results of these studies are the collapse of the spin gap [14,16], and the competition between the SP and the Néel states 14 17], when a small amount of impurities is introduced. From the theoretical point of view, the introduction of non-magnetic impurities, which for the nearest-neighbors Heisenberg model leads to the appearance of chains with open boundary conditions (OBC), has been analyzed using conformal field theory and Monte Carlo simulations [19,20]. The main result of these studies is the appearance of enhanced staggered spin order, which is maximized near the vacancies. More recently, it has been shown that spin-1/2 states are localized near vacancies in dimerized chains and other systems [21 23]. The experimentally observed stabilization of a Néel state upon $\mathrm{Zn}$ doping in dimerized chains can be related to the enhancement of antiferromagnetic (AF) correlations near the vacancies which arises due to the presence of these bound states. The effect has been studied theoretically using both analytical [24] and numerical techniques [21,22]. These bound states are expected to produce spectral weight inside the original gap [25], and there is already direct experimental evidence compatible with the existence of these low-energy excitations [26,27. Results very similar to those obtained in dimerized chains 
have also been obtained in ladder systems both experimentally [28] and theoretically [21,22, 25, 29].

In the present article, the previous studies performed on $1 \mathrm{D}$ AF spin-1/2 dimerized Heisenberg chains [21,22,25] are extended to the case of magnetic impurities, in particular spin-1 impurities. In previous literature, it has been studied the related case of spin-1 AF Heisenberg chains doped with non-magnetic impurities which become, again, equivalent to chains with OBC 30,31. The most important prediction in this context is the presence of isolated spin- $1 / 2$ states on the chain edges. Experimentally, magnetization and electron spin resonance studies on the compound $\mathrm{Ni}\left(\mathrm{C}_{3} \mathrm{H}_{10} \mathrm{~N}_{2}\right)_{2} \mathrm{~N}_{3}\left(\mathrm{ClO}_{4}\right)$ have confirmed the existence of these end-chain spin-1/2 spins [32]. The end-states are easily explained by the valence bond solid (VBS) picture of the ground state of spin-1 Haldane chains [33]. In the same spirit, an unifying picture based on a short-range resonant-valence-bond (RVB) scenario 34, 35, has been formulated to explain the existence of spin- $1 / 2$ bound states in gapped spin- $1 / 2$ systems, as discussed in the previous paragraph 21,22].

In the present study of spin- $1 / 2$ dimerized chains doped with spin-1 impurities, both RVB and VBS pictures will be used to interpret our results which were obtained using Lanczos diagonalization and quantum Monte Carlo (QMC) simulations. Our main conclusion is that the overall effects, such as the destruction of the spin gap by low-energy excitations due to bound states near the impurities, are similar to those generated by nonmagnetic impurities in spite of the fact that the microscopic origin and details of these bound states are somewhat different. This difference manifests in a more localized enhancement of the AF correlations in the immediate vicinity of the $S=1$ impurity than in the case of nonmagnetic impurities. However, at distances beyond one lattice spacing away from the $S=1$ impurity the spin-spin correlations behave very similarly to the case of doped nonmagnetic impurities. Thus, globally magnetic and nonmagnetic impurities apparently lead to similar physics including enhanced antiferromagnetic correlations.

We wish to emphasize that in this article we are mainly concerned with dimerized chains, containing only spin degrees of freedom, and which can be realized in nature independently of the spin-Peierls mechanism. The spinPeierls transitions recently found in inorganic materials only add an extra motivation to study dimerized chains since they can describe reasonably well certain features of these materials at very low temperatures. To properly describe some other features, the inclusion of phononic degrees of freedom is strictly necessary at least at the mean field level 36 38 (see e.g., discussion in Section IV). In this sense, our purpose is to give just indications that some of the results we found could be related to some experimental findings in spin-Peierls compounds. It is quite likely that similar experimental studies are going to be carried on in the near future on other materials containing dimerized chains.

The paper is organized as follows. In Section III the strength of the effective Heisenberg interaction between a $\mathrm{Cu}$-ion and an impurity Ni-ion is computed starting from a multiband Hubbard model. In Section III, the dynamic spin structure factor is studied in order to analyze the suppression of the spin gap in a dimerized chain by the introduction of spin- 1 impurities. The main differences with respect to the case of nonmagnetic impurities are studied. The spin-spin correlations near the impurities have also been investigated in order to evaluate the strength of the AF order enhancement. In Section IV], results for the magnetic susceptibility obtained by quantum Monte Carlo simulations are presented. Finally, in the Conclusions section, the most important results are discussed, together with their interpretation within a simple valence bond picture. Experimental consequences of our calculations are here also discussed.

\section{EFFECTIVE INTERACTION}

Before addressing the spin chain model and computational techniques used in this paper, it is important to estimate the coupling between the $S=1$ impurity and neighboring $S=1 / 2$ ions. In previous studies based on $\mathrm{Zn}$-doping, this calculation was not necessary since $\mathrm{Zn}^{2+}$ has zero spin. However, for the case of $\mathrm{Ni}^{2+}$ impurities with $S=1$, a Heisenberg coupling between $\mathrm{Ni}$ and $\mathrm{Cu}$ should be part of any model that attempts to describe their behavior. In this section a rough estimation of the nickel-copper exchange will be made. The calculation will be carried out for the case of a $180^{\circ} \mathrm{Cu}-\mathrm{O}-\mathrm{Ni}$ bond, which is relevant for a variety of materials such as the $1 \mathrm{D}$ compounds $\mathrm{Sr}_{2} \mathrm{CuO}_{3}$ and $\mathrm{SrCuO}_{2}$, the $\mathrm{Cu}$-based ladder systems mentioned in the previous section, the High-Tc cuprates, and several others. The results are intended to provide just qualitative information about the strength of the exchange. In principle, they do not apply directly to systems such as $\mathrm{CuGeO}_{3}$ where the $\mathrm{Cu}$-ions are linked by two oxygens with $\sim 90^{\circ}$ bonds. Carrying out a calculation for this particular material would be difficult because small exchange couplings are expected to emerge from a problem involving large energy scales of the order of eV, and the rough one-bond calculation discussed below may not be enough to produce reliable results.

The Hamiltonian analyzed in this section is given by $H=H_{t}+H_{V}+H_{H}$, with the hopping term defined as

$$
\begin{aligned}
H_{t}= & -\sum_{\sigma} t_{C u O}\left(c_{C u, \sigma}^{\dagger} c_{O, \sigma}+\text { h.c. }\right) \\
& -\sum_{\sigma, \alpha} t_{N i O \alpha}\left(c_{N i \alpha, \sigma}^{\dagger} c_{O \alpha, \sigma}+\text { h.c. }\right),
\end{aligned}
$$

where $\sigma$ denotes the spin, and $\alpha=1,2$ the two active orbitals of $\mathrm{Ni}$ (labeled as Ni1 and Ni2). $c_{C u, \sigma}$ and $c_{N i \alpha, \sigma}$ are destruction operators for fermions on the $\mathrm{Cu}$ and $\mathrm{Ni} \alpha$ 
orbitals, respectively. $t_{\mathrm{CuO}}\left(t_{\mathrm{NiO \alpha}}\right)$ is the hopping amplitude for fermions to move between $\mathrm{Cu}$ and $\mathrm{O}$ (Ni $\alpha$ and $\mathrm{O})$. The rest of the notation is standard. The interaction $H_{V}$ is defined as

$$
\begin{aligned}
H_{V} & =U_{C u} n_{C u, \uparrow} n_{C u, \downarrow}+U_{O} n_{O, \uparrow} n_{O, \downarrow} \\
& +U_{N i} \sum_{\alpha} n_{N i \alpha, \uparrow} n_{N i \alpha, \downarrow} \\
& -\Delta_{C u} n_{C u}-\Delta_{N i} \sum_{\alpha} n_{N i \alpha},
\end{aligned}
$$

where $n_{C u, \sigma}$ is the number operator at the $C u$ site with spin $\sigma, n_{C u}=n_{C u, \uparrow}+n_{C u, \downarrow}$, and the notation for $\mathrm{Ni}$ and $\mathrm{O}$ follows analogous definitions. $U_{C u}, U_{O}, U_{N i}$ regulate the on-site Coulomb repulsion for $\mathrm{Cu}, \mathrm{O}$, and $\mathrm{Ni}$, respectively. $\Delta_{C u}$ is the energy needed to move a fermion from $\mathrm{Cu}$ to $\mathrm{O}$, while $\Delta_{N i}$ is a similar quantity but to move fermions from the two $N i$ orbitals (assumed degenerate) to O. Finally,

$$
H_{H}=-J_{H} \mathbf{S}_{1} \cdot \mathbf{S}_{2}
$$

is the ferromagnetic Hund coupling $\left(J_{H}>0\right)$ between the two active orbitals at the Ni site (with $\mathbf{S}_{\alpha}$ being the spin at orbital $\alpha$ ).

This Hamiltonian can be solved exactly for the case of interest that contains 3 electrons, two of which are expected to be mostly at the Ni-ion and the other at the $\mathrm{Cu}$-ion. From the difference in energy between the ground state and the first excited state with spin 1 in the spectrum the exchange coupling can be calculated. The matrix that needs to be diagonalized is $24 \times 24$.

The parameters corresponding to the $\mathrm{Cu}$ and $\mathrm{O}$ ions are approximately known from previous theoretical and experimental work in the context of high-temperature superconductors. The actual parameter values used here are 39] $\Delta_{C u} \sim 3.6 \mathrm{eV}, t_{C u O} \sim 1.3 \mathrm{eV}, U_{O} \sim 4.6 \mathrm{eV}$, and $U_{C u} \sim 10.5 \mathrm{eV}$. The rest of the parameters related with Ni-oxides were discussed in previous work by two of the present authors and collaborators [40], in the context of hole doped $S=1$ chains. In that publication it was argued that $\Delta_{N i} \sim 6.0 \mathrm{eV}, U_{N i} \sim 9.5 \mathrm{eV}, t_{N i O 1} \sim 1.3 \mathrm{eV}$, $t_{\mathrm{NiO} 2} \sim 0.75 \mathrm{eV}$, and $J_{H} \sim 1.3 \mathrm{eV}$, based on a variety of experimental work and some cluster calculations. These will be the parameters used in the present study.

As a warm-up, the similar cases of $180^{\circ} \mathrm{Cu}-\mathrm{O}-\mathrm{Cu}$ and $\mathrm{Ni}-\mathrm{O}-\mathrm{Ni}$ bonds were studied using the same method, since the exchanges for $\mathrm{CuO}_{2}$ and $\mathrm{NiO}_{2}$ planes are known experimentally and, thus, a comparison between the computational results and properties of the real materials can be carried out. The Hamiltonian parameters used are the same as given before. For the $\mathrm{Cu}-\mathrm{O}-\mathrm{Cu}(\mathrm{Ni}-\mathrm{O}-$ $\mathrm{Ni}$ ) bond the size of the matrix to diagonalize is $9 \times 9$ $(100 \times 100)$ for the case of two (four) fermions in the problem. For Ni-O-Ni, four fermions are needed to represent the two expected spin-1 states in this bond. For the copper oxides, the exact diagonalization of the Hamiltonian matrix obtained from Eqs.(1-3) leads to an exchange
$J_{C u-O-C u}=(100 \pm 30) m e V$, while for the nickel oxides it is $J_{N i-O-N i}=(20 \pm 10) m e V$. The error bars are crudely estimated as follows: 1 . the many parameters entering the Hamiltonian were arbitrarily changed one by one by $\pm 20 \%$ (to simulate uncertainties in their experimental determination), and in each case the new value of $J$ was calculated, 2. a histogram with the distribution of $J$ 's was built, and from a fit of these results with a Gaussian function, the error bars were determined as the width of such a Gaussian. It was found that the parameters that affect the exchange the most are the hopping amplitudes and $\Delta_{C u}$. The Coulomb energies and Hund coupling are large enough such that small variations in their values do not affect quantitatively the results.

Considering the roughness of the calculation, it is gratifying that the theoretically calculated Heisenberg couplings presented above are in very good agreement with the experimental results for both materials (see for instance Ref. 41]). Thus, it is expected that the theoretical prediction for $J_{N i-O}-C u$ will be at least qualitatively correct as well. The result obtained in this paper is $J_{N i-O-C u}=(100 \pm 50) m e V$ i.e. very similar to the case of copper-oxides. Then, it is here estimated that using a spin model with the same exchange $J$ among the $S=1 / 2$ spins, and among these spins and the $S=1 \mathrm{im}-$ purity, represents a good approximation to the problem investigated in this paper.

\section{DYNAMICAL STRUCTURE FACTOR AND SPIN-SPIN CORRELATIONS.}

The one-dimensional model which describes a spin- $1 / 2$ Heisenberg chain in the presence of spin- 1 impurities is defined as:

$$
\begin{array}{r}
\mathcal{H}=J \sum_{i \neq i_{0}, i_{0}-1}\left(1+\delta_{i}\right) \mathbf{S}_{i} \cdot \mathbf{S}_{i+1} \\
+J^{\prime} \sum_{i_{0}}\left\{\left(1+\delta_{i_{0}-1}\right) \mathbf{S}_{i_{0}-1} \cdot \mathbf{s}_{i_{0}}\right. \\
\left.\quad+\left(1+\delta_{i_{0}}\right) \mathbf{s}_{i_{0}} \cdot \mathbf{S}_{i_{0}+1}\right\}
\end{array}
$$

where $\mathbf{S}_{i}$ denote the spin-1/2 degrees of freedom, and $\mathbf{s}_{i_{0}}$ are the spin-1 operators located at the impurity sites $i_{0}$. The dimerization is staggered and it corresponds to $\delta_{i}=(-1)^{i} \delta$. Periodic boundary conditions are imposed unless otherwise stated. According to the results of the previous section, in the following the case $J^{\prime}=J$ will be considered. Note that in Hamiltonian Eq.(4) an interaction when two spin-1 impurities are located next to each other has not been included due to the result of the previous section suggesting that $J_{N i-O-N i} \ll J_{C u-O-C u}, J_{C u-O-N i}$.

Using Lanczos diagonalization and the standard continued fraction formalism [39], the zero temperature spin-spin correlations and dynamical structure factor for model Eq.(4) have been computed. In order to analyze 
finite-temperature properties a world-line Monte Carlo algorithm [42] suitable for the present problem has been implemented. This method has allowed us to reach low enough temperatures such that the ground state behavior of spin-spin correlations can be calculated. Thus, with this technique it is possible to treat longer chains than those accessible to the Lanczos method, having only a small finite-temperature contamination.

\section{A. One impurity}

Let us first investigate whether a suppression of the spin gap occurs when $\mathrm{S}=1$ impurities are introduced in a dimerized chain, similarly as it occurs in Zn-doped systems. The analysis here follows closely the study performed in Ref. 25] for the case of nonmagnetic impurities. Since (i) the spin gap opens at $k=\pi$, and (ii) the most intense peak of the dynamical structure function $S(k, \omega)$ also appears at $k=\pi$, in agreement with neutron scattering experiments, then the present analysis starts by studying the behavior of $S(\pi, \omega)$ upon the introduction of $S=1$ impurities.

The dynamical structure factor at zero temperature is defined by:

$S^{z z}(k, \omega)=\frac{1}{N} \sum_{n}\left|\left\langle\psi_{n}\left|S^{z}(k)\right| \psi_{0}\right\rangle\right|^{2} \delta\left(\omega-\left(E_{n}-E_{0}\right)\right)$,

where $S^{z}(k)=(1 / N) \sum_{m} S_{m}^{z} e^{i k m}, N$ is the number of sites, $m$ labels the sites of the lattice, and the rest of the notation is standard. In the case of total dimerization, i.e. $\delta=1$, it can be easily deduced that the introduction of a single impurity generates a peak in $S(\pi, \omega)$ inside the gap, actually at $\omega=0$. This is due to the fact that the matrix element $M=\left\langle\eta_{0}\left|S^{z}(k)\right| \eta_{0}\right\rangle=(1 / 3)\left(2-(1 / 2) e^{i k}\right)$ is nonzero. Here $\left|\eta_{0}\right\rangle$ is the ground state of the 2 -site dimer that carries the impurity i.e. $\left|\eta_{0}\right\rangle=\frac{1}{\sqrt{3}}(|0, \uparrow\rangle-$ $\sqrt{2}|1, \downarrow\rangle)$, where $1,0,-1$ label the states of the spin-1, and $\uparrow, \downarrow$ correspond to the spin- $1 / 2$. This simple mechanism is also present for impurities of higher spin such as Co, 43] and actually includes $\mathrm{Zn}$ as a special case. The important point here is that in the bond being doped a state with a total nonzero spin appears after a spin- $1 / 2$ is replaced by a spin different from $1 / 2$. This nonzero spin in the dimer where the doping occurs is decoupled from the rest of the lattice in the extreme limit $\delta=1$ and, thus, produces weight at zero energy in the dynamical spin structure factor.

\section{B. Two Impurities}

Let us now consider the effect of two impurities located at arbitrary distances along a chain. If two impurities are located in a periodic chain of $N$ sites, it is also easy to understand in the case of total dimerization $\delta=1$ (and remembering that the ground state is selected to have a total spin projection on the $z$-axis equal to zero to mimic the case of $\delta<1$ ) that if the first impurity is located at site 1 then a spectral weight at $\omega=0$ appears only if the second impurity is located at sites $l_{2}$ which are even. For instance, if $l_{2}$ is 2 then two free spin- 1 are located in the same dimer which naturally will produce weight at zero frequency (remember that the $\mathrm{Ni}-\mathrm{Ni}$ exchange has been neglected here since it is much smaller than the $\mathrm{Cu}-\mathrm{Cu}$ or $\mathrm{Cu}-\mathrm{Ni}$ exchanges). However, if $l_{2}=3$, then the matrix element $M$ mentioned in the previous paragraph will have opposite signs for the dimers involving sites 1-2 and 3-4 (note again that the total spin of the global
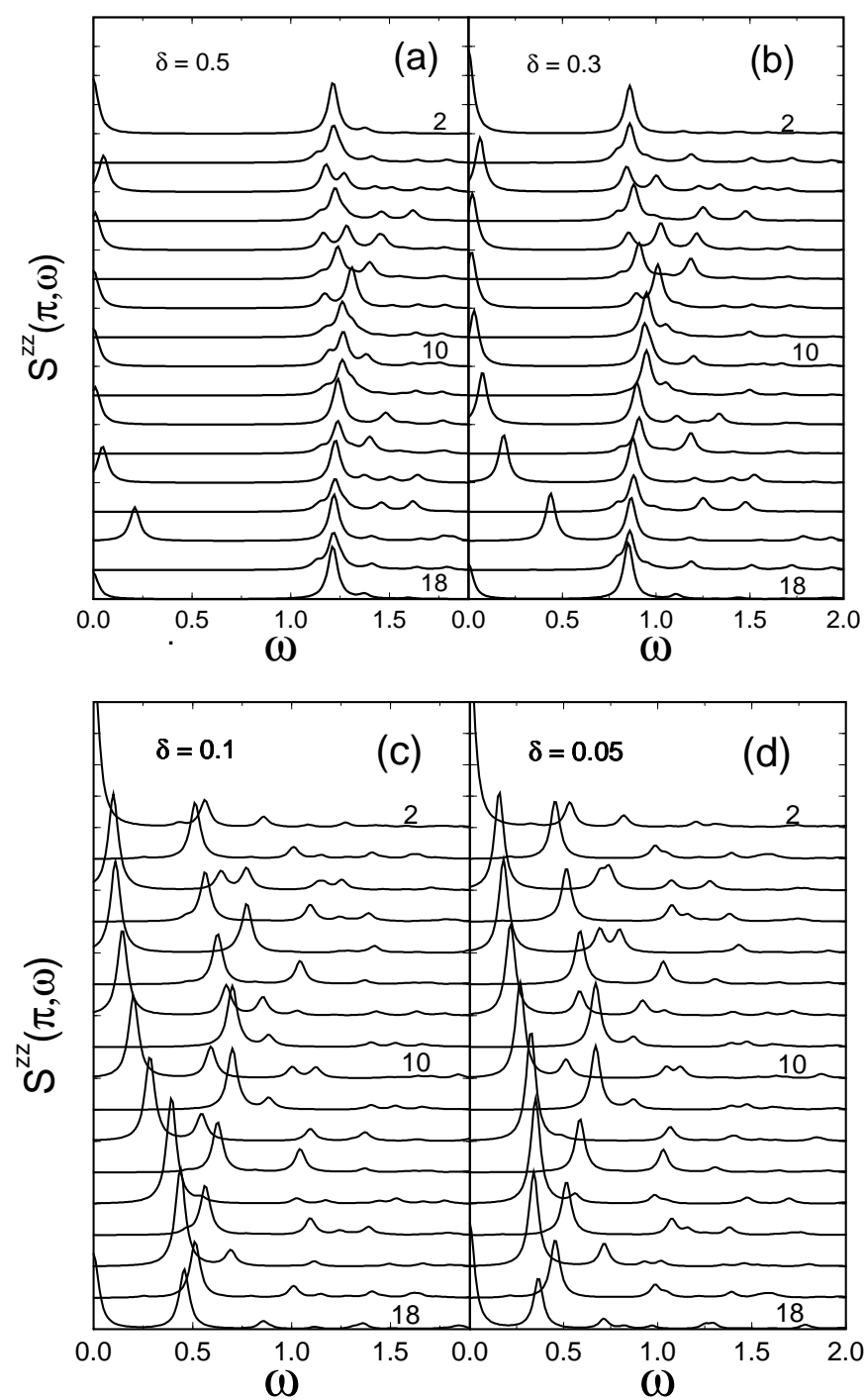

FIG. 1. Dynamical structure factor at $k=\pi$ obtained by exact diagonalization using a 18 site chain with two $S=1$ impurities, total $S^{z}=0$, as a function of the position of the second impurity and for several values of $\delta$. One of the impurities is located at site 1 , and the position of the second impurity is indicated increasing from top to bottom.

state is zero), and thus the weight of the zero-energy peak 
cancels. A similar argument holds for $l_{2}$ equal to any odd-integer. On the other hand, for $l_{2}$ even-integers the matrix elements of the two doped bonds contribute with the same sign and a low-energy peak should appear in the spectrum. These results deduced at large $\delta$ actually are qualitatively similar when $\delta$ is reduced from 1.0, as it can be observed in Fig. 1 1 where $S^{z z}(\pi, \omega)$ obtained for $N=18$ is shown. In this figure, it is clear that even for $\delta$ as small as 0.05 a substantial weight inside the gap is present only when the second impurity is located in evensites, in agreement with the discussion of the previous paragraph. In this figure, the bond between the sites 1 and 2 is "strong" $\left(J_{i}=J(1+\delta)\right)$. As $\delta$ decreases the positions of the impurity peaks inside the gap shift to larger values of $\omega$ and increase their weight, as expected.

In Fig. 2, $S^{z z}(\pi, \omega)$ for $N=18$ without impurities and in the presence of two $S=1$ impurities for several values of $\delta$ is presented. For this result an average over all the impurity positions has been carried out. In this figure the corresponding quantity for nonmagnetic impurities is also included for comparison. It can be observed that the weight which appears inside the original gap of the pure system is consistingly larger for $S=1$ impurities than for nonmagnetic impurities. This weight inside the gap in the case of $S=1$ impurities may correspond to the bound states formed between the spin-1, considered as two $S=$ $1 / 2$ spins as in the VBS picture, and the two neighboring spin- $1 / 2$ spins. It is also reasonable to assume that the weight of these bound states in the ground state is larger than the weight of the bound states due to the presence of $\mathrm{OBC}$, at least in the RVB picture of the ground state discussed below, thus explaining the behavior shown in Fig. 2.

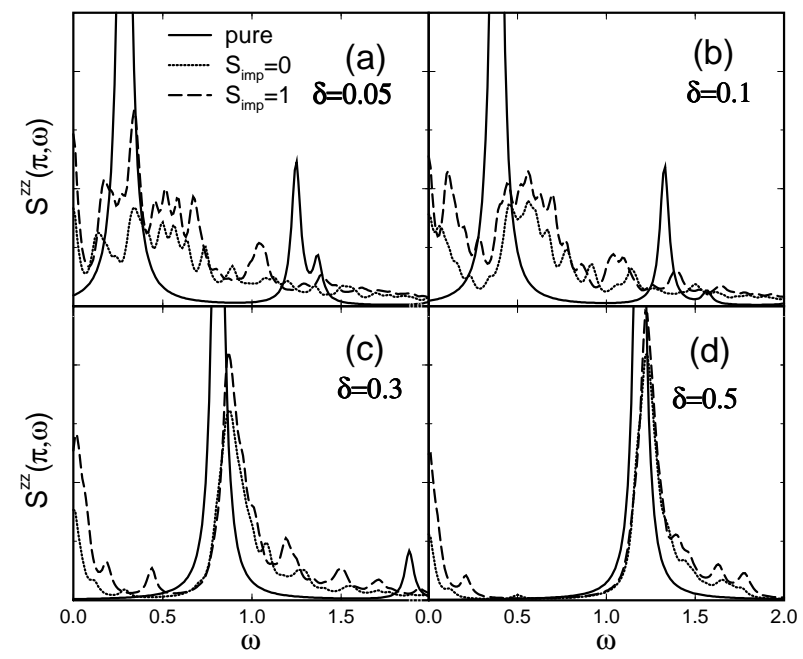

FIG. 2. Dynamical structure factor at $k=\pi$ obtained by exact diagonalization in the 18 site chain for the pure system, with two $S=1$ impurities, and with two nonmagnetic impurities, total $S^{z}=0$, for several values of $\delta$. These curves have been obtained by averaging over all impurity positions.

\section{Enhanced Spin Correlations Near Ni}

It is also instructive to analyze from a microscopic point of view the effects of a spin-1 impurity on the local magnetic order. This can be achieved by studying the spin-spin correlations around the impurity defined as $S(l)=<S^{z}\left(i_{1}\right) S^{z}\left(i_{1}+l\right)>$, where $i_{1}$ is the site next to the impurity located at $i_{0}$, and $l \ll N$. A normalization such that $S(0)=1$ was adopted. To predict the behavior of this correlation it is possible to invoke the short-range RVB picture of the ground state of spin- $1 / 2$ $1 \mathrm{D}$ chains previously used to describe the effect of nonmagnetic impurities 22,25. In this picture, the ground state is assumed to be dominated by the superposition of the two possible dimerized states, one with singlets on the odd bonds and the other with singlets on the even bonds. In the case of nonmagnetic impurities, a simple argument shows that the correlation $S(l)$ is enhanced near the impurities with respect to the same correlations for the pure system. The idea is simply that without vacancies a given spin spends roughly half the time coupled into singlets with each of its two neighbors, while when the spin being studied is located next to a vacancy only one neighbor is available for singlet formation and naturally the correlation between these two spins is enhanced. The argument can be extended to distances larger than one lattice spacing easily 21].

By using this same RVB picture for the case of spin1 impurities one obtains, however, a somewhat different behavior. In the first place let us observe that the energy of a dimer formed by a spin- 1 and a spin- $1 / 2$ is $-J_{i}$, whereas the energy of a "pure" dimer made out of two spin- $1 / 2$ has energy $-3 J_{i} / 4$. Then, the spin- $1 / 2$ next to the impurity should have a stronger tendency to form a singlet with the impurity rather than with other spin$1 / 2$ sites, simply to minimize the energy. In addition, and most importantly, is that the ground state energy of the three site cluster formed by the impurity and its two spin- $1 / 2$ neighbors is equal to $(-2 J)$, independent of $\delta$. If one considers a subsystem with four sites containing the $S=1$ impurity, it can be deduced that in order to minimize the energy this subsystem prefers to form a three-site cluster leaving a spin-1/2 unpaired rather than two dimers, one of them containing the impurity. This is true at least for $\delta<1 / 7$ if the two dimers are formed on "strong" bonds, and for all $\delta>0$ if they correspond to "weak" bonds. Thus, it is expected that states containing the ground state of this three-site 1/2-1-1/2 system, which corresponds to a singlet, plus an unpaired spin and a RVB configuration for the rest of the chain will have a large overlap with the actual ground state of the problem. The coefficients of the states in the three-site system ground state favors the AF correlations between the three sites. These two factors imply that: 1 . there is a strong AF correlation between a site next to the impurity and the impurity itself, $S_{i m p}(1), 2$. there is an enhancement of the correlation between both sites next 
to the impurity, $S_{\text {cross }}(2)$, and 3 . there is a reduction of the correlations between site $i_{1}$ (the site next to the impurity) and the rest of the spins located on the same side with respect to the impurity. In summary, what is predicted is a very local enhancement of the correlations around the spin-1 impurity. The region involved in the process corresponds to only 3 spins (the spin- 1 and the two neighboring spin-1/2). From the point of view of the rest of the spins on the chain, this three-spin structure has formed a singlet and it behaves effectively as a nonmagnetic vacancy. Thus, this result for dimerized chains agrees with a general result obtained for uniform AF Heisenberg chains which states that an impurity with spin-S is equivalent to open boundary conditions with a decoupled spin of size (S-1). 19 Hence, a spin-1 impurity is equivalent to a non-magnetic impurity and forms a singlet.

These features can be clearly seen in Table If. The correlations $S(l)$ have been computed with a standard world-line Monte Carlo at a temperature $T=0.0625 \mathrm{~J}$ and for $N=80$, except otherwise stated. The dimerization is $\delta=0.05$. The statistical error of the QMC simulations affects only the last digit of the quantities reported. The effects of nonmagnetic impurities are shown for the 40 site chains for the case where OBC are used. Results for the 39 site chains are very similar to the values for the 40 site chain. The effects of $S=1$ impurities are shown in the last four rows. In these cases the position of the second impurity $i_{0}$ is shown (the first one is located at site 1). In all cases, it has been indicated if the bond between the reference site, $i_{1}$, and its spin- $1 / 2$ nearest neighbor site is strong (s) or weak (w). In Table II, the dependence of the previous results with $\delta$ is studied using Lanczos diagonalization results for a chain with $N=20$ sites and one impurity. Note the fairly good agreement between these zero temperature results compared with the finite temperature QMC results on the 80 site chain both for the pure system and for the case of two impurities located at sites $i_{0}=1,41$ and $\delta=0.05$. It can be observed on Table II that as $\delta$ is increased from 0.05 to 0.5 there is a smooth variation in the behavior of the various correlations shown. In all cases $S(1), S(2)$ and $S(3)$ are reduced from their pure values, and $S_{i m p}(1)$ and $S_{\text {cross }}(2)$ are enhanced.

The possibility raised above that the singlet ground state of the three-site subsystem $1 / 2-1-1 / 2$ could behave as an effective nonmagnetic vacancy, and hence that it may lead to an enhancement of the AF correlations 21,22 between the sites close to this vacancy, is actually confirmed by the results shown in Table III. In this Table, the correlations between a spin next to one of these three-site clusters, i.e. located at two lattice spacings from the $S=1$ impurity, and the remaining spins of the chain are shown to be enhanced with respect to their values in the pure system. As previously noticed [21,22], the enhancement of the correlations for the case when the bond between the two first sites next to a vacancy is weak is more pronounced than the case of a strong bond between them.

TABLE I. Spin-spin correlations $S(l)$ at various distances as defined in the text obtained by Monte Carlo simulations on chains with $N=80$ sites, using periodic boundary conditions, and at $T=0.0625$ unless otherwise stated. "Pure" means that there are no impurities. The chains with $N=39,40$ sites have open boundary conditions, and the correlations are measured starting at the site next to the boundary. The dimerization is $\delta=0.05$. The last four rows correspond to the chain in the presence of two $S=1$ impurities, the first one located at site 1 and the second one at site $i_{0}$. The reference site is located at $i_{1}$. $w, s$ refer to weak and strong bonds, respectively, between the spin at site $i_{1}$ and its spin-1/2 neighbor.

\begin{tabular}{|c|c|c|c|c|c|}
\hline \hline & $\mathrm{S}(1)$ & $\mathrm{S}(2)$ & $\mathrm{S}(3)$ & $\mathrm{S}_{\text {cross }}(2)$ & $\mathrm{S}_{\text {imp }}(1)$ \\
\hline pure s & -0.777 & 0.230 & -0.247 & - & - \\
\hline pure s $(\mathrm{T}=0.05)$ & -0.785 & 0.230 & -0.250 & - & - \\
\hline pure w & -0.400 & 0.230 & -0.122 & - & - \\
\hline pure w $(\mathrm{T}=0.05)$ & -0.408 & 0.230 & -0.126 & - & - \\
\hline $\mathrm{N}=40 \mathrm{~s}$ & -0.906 & 0.242 & -0.274 & - & - \\
\hline $\mathrm{N}=40 \mathrm{~s}(\mathrm{~T}=0.05)$ & -0.907 & 0.244 & -0.275 & - & - \\
\hline $\mathrm{N}=40 \mathrm{w}$ & -0.777 & 0.297 & -0.253 & - & - \\
\hline $\mathrm{N}=40 \mathrm{w}(\mathrm{T}=0.05)$ & -0.777 & 0.299 & -0.254 & - & - \\
\hline imp. $\mathrm{i}_{0}=40 \mathrm{i}_{1}=41 \mathrm{~s}$ & -0.408 & 0.170 & -0.097 & 0.295 & -0.971 \\
\hline imp. $\mathrm{i}_{0}=40 \mathrm{i}_{1}=39 \mathrm{w}$ & -0.313 & 0.189 & -0.081 & 0.295 & -1.067 \\
\hline imp. $\mathrm{i}_{0}=41 \mathrm{i}_{1}=40 \mathrm{~s}$ & -0.429 & 0.196 & -0.141 & 0.293 & -1.148 \\
\hline imp. $\mathrm{i}_{0}=41 \mathrm{i}_{1}=42 \mathrm{w}$ & -0.293 & 0.193 & -0.087 & 0.293 & -1.240 \\
\hline \hline
\end{tabular}

TABLE II. Spin-spin correlations at various distances as defined in the text obtained by Lanczos diagonalization for $N=20$, using periodic boundary conditions and for several values of the dimerization $\delta$.

\begin{tabular}{|c|c|c|c|c|c|}
\hline \hline & $\mathrm{S}(1)$ & $\mathrm{S}(2)$ & $\mathrm{S}(3)$ & $\mathrm{S}_{\text {cross }}(2)$ & $\mathrm{S}_{\text {imp }}(1)$ \\
\hline pure w $(\delta=0.05)$ & -0.4113 & 0.2319 & -0.1339 & - & - \\
\hline pure w $(\delta=0.1)$ & -0.3213 & 0.2111 & -0.0927 & - & - \\
\hline pure w $(\delta=0.3)$ & -0.1706 & 0.1401 & -0.0329 & - & - \\
\hline pure w $(\delta=0.5)$ & -0.0954 & 0.0860 & -0.0117 & - & - \\
\hline pure s $(\delta=0.05)$ & -0.7679 & 0.2319 & -0.2466 & - & - \\
\hline pure s $(\delta=0.1)$ & -0.8459 & 0.2111 & -0.2378 & - & - \\
\hline pure s $(\delta=0.3)$ & -0.9503 & 0.1401 & -0.1574 & - & - \\
\hline pure s $(\delta=0.5)$ & -0.9834 & 0.0860 & -0.0931 & - & - \\
\hline $\mathrm{w}(\delta=0.05)$ & -0.3012 & 0.1953 & -0.0976 & 0.2936 & -1.2385 \\
\hline $\mathrm{w}(\delta=0.1)$ & -0.2579 & 0.1828 & -0.0760 & 0.2838 & -1.2600 \\
\hline $\mathrm{w}(\delta=0.3)$ & -0.1511 & 0.1272 & -0.0300 & 0.2179 & -1.3018 \\
\hline $\mathrm{w}(\delta=0.5)$ & -0.0870 & 0.0795 & -0.0110 & 0.1408 & -1.3211 \\
\hline $\mathrm{s}(\delta=0.05)$ & -0.4325 & 0.1951 & -0.1438 & 0.2936 & -1.1462 \\
\hline $\mathrm{s}(\delta=0.1)$ & -0.5135 & 0.1832 & -0.1569 & 0.2838 & -1.0723 \\
\hline $\mathrm{s}(\delta=0.3)$ & -0.7789 & 0.1315 & -0.1396 & 0.2179 & -0.7346 \\
\hline $\mathrm{s}(\delta=0.5)$ & -0.9206 & 0.0841 & -0.0901 & 0.1408 & -0.4342 \\
\hline \hline
\end{tabular}


TABLE

III.

Spin-spin correlations $S(2, l)=\left\langle S^{z}(2) S^{z}(2+l)\right\rangle$ at various distances from a site two lattice spacings apart from a $S=1$ impurity obtained by Lanczos diagonalization for $N=20$ for various values of $\delta$, and by Monte Carlo simulations for $N=80$ sites and $\delta=0.05$.

\begin{tabular}{|c|r|r|r|r|}
\hline \hline & $\mathrm{S}(2,3)$ & $\mathrm{S}(2,4)$ & $\mathrm{S}(2,5)$ & $\mathrm{S}(2,6)$ \\
\hline pure s $(\delta=0.05)$ & -0.7679 & 0.2319 & -0.2466 & 0.1243 \\
\hline pure s $\delta=0.10)$ & -0.8459 & 0.2111 & -0.2378 & 0.0960 \\
\hline pure s $\delta=0.30)$ & -0.9503 & 0.1401 & -0.1574 & 0.0360 \\
\hline pure s $(\delta=0.50)$ & -0.9834 & 0.0860 & -0.0931 & 0.0125 \\
\hline $\mathrm{s}(\delta=0.05)$ & -0.8224 & 0.2371 & -0.2580 & 0.1255 \\
\hline $\mathrm{s}(\delta=0.10)$ & -0.8696 & 0.2141 & -0.2421 & 0.0976 \\
\hline $\mathrm{s}(\delta=0.30)$ & -0.9531 & 0.1404 & -0.1577 & 0.0361 \\
\hline $\mathrm{s}(\delta=0.50)$ & -0.9838 & 0.0861 & -0.0931 & 0.0125 \\
\hline pure w $(\delta=0.05)$ & -0.4113 & 0.2319 & -0.1339 & 0.1243 \\
\hline pure w $(\delta=0.10)$ & -0.3213 & 0.2111 & -0.0927 & 0.0960 \\
\hline pure w $(\delta=0.30)$ & -0.1706 & 0.1401 & -0.0329 & 0.0360 \\
\hline pure w $(\delta=0.50)$ & -0.0954 & 0.0860 & -0.0117 & 0.0125 \\
\hline $\mathrm{w}(\delta=0.05)$ & -0.6263 & 0.2708 & -0.2113 & 0.1602 \\
\hline $\mathrm{w}(\delta=0.10)$ & -0.5069 & 0.2633 & -0.1606 & 0.1417 \\
\hline $\mathrm{w}(\delta=0.30)$ & -0.2183 & 0.1678 & -0.0464 & 0.0492 \\
\hline $\mathrm{w}(\delta=0.50)$ & -0.1040 & 0.0926 & -0.0133 & 0.0141 \\
\hline $\mathrm{N}=80$ pure s & -0.777 & 0.230 & -0.247 & 0.115 \\
\hline $\mathrm{N}=80$ pure w & -0.400 & 0.230 & -0.122 & 0.116 \\
\hline $\mathrm{N}=80 \mathrm{i}_{0}=40 \mathrm{~s}$ & -0.815 & 0.231 & -0.251 & 0.117 \\
\hline $\mathrm{N}=80 \mathrm{i}_{0}=40 \mathrm{w}$ & -0.619 & 0.260 & -0.200 & 0.143 \\
\hline $\mathrm{N}=80 \mathrm{i}_{0}=41 \mathrm{~s}$ & -0.829 & 0.235 & -0.260 & 0.120 \\
\hline $\mathrm{N}=80 \mathrm{i}_{0}=41 \mathrm{w}$ & -0.629 & 0.271 & -0.210 & 0.157 \\
\hline \hline
\end{tabular}

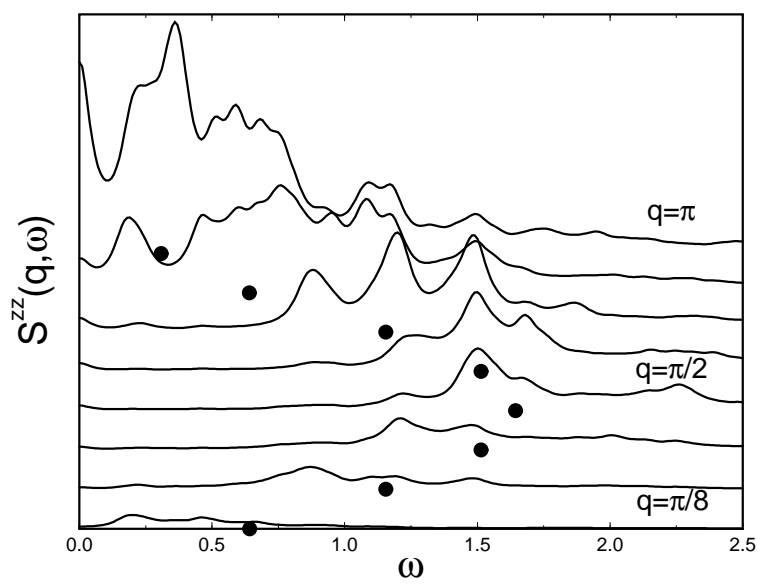

FIG. 3. Dynamical structure factor $S^{z z}(q, \omega)$ obtained by exact diagonalization in the 16 site chain with two $S=1 \mathrm{im}$ purities for $\delta=0.05$ averaged over all the impurity positions. The energy of the first peak for the pure $N=16$ chain are indicated with full circles.
In summary, the results shown thus far seem to indicate that for magnetic impurities the AF correlations between these impurities and their two neighboring spin$1 / 2$ 's are strong. However, note that once the three-spin cluster centered at the impurity is considered as a singlet, then the rest of the spins will enhance the correlations among themselves as if in the presence of a nonmagnetic impurity.

Finally, in Fig. $3 S^{z z}(k, \omega)$ is shown for $N=16$, $\delta=0.05$ and in the presence of two $S=1$ impurities. The results are averaged over all the impurity positions. The energies of the first peaks for the pure system have been indicated for reference. The behavior of $S^{z z}(k, \omega)$ in the presence of $S=1$ impurities is very similar to the previously reported behavior for nonmagnetic impurities [25]. In other words, two bands appear here: 1. the low energy "impurity band" with the highest intensity peaks at or near $k=\pi$, and 2. a high energy band, which is essentially a remnant of the dispersion relation for the undoped system.

\section{MAGNETIC SUSCEPTIBILITY AT FINITE TEMPERATURES}

The suppression of the spin gap due to magnetic impurities can also be detected in thermodynamical properties such as the magnetic susceptibility $\chi(\mathrm{T})$. It is well-known that the presence of a spin gap between the singlet ground state and the excited triplet state leads to an exponential decay as the temperature is reduced to zero. On the other hand, $\chi(\mathrm{T})$ would diverge or converge to a nonzero value as $\mathrm{T}$ vanishes for a gapless system, such as the uniform 1D Heisenberg model. A similar result should emerge as a consequence of the presence of states inside the original gap induced by impurities, as discussed in the previous section. This change of the magnetic susceptibility upon $\mathrm{Zn}, \mathrm{Mg}$ or $\mathrm{Ni}$ doping was indeed observed experimentally in the SP compound $\mathrm{CuGeO}_{3}$. 14, 16, 44 In particular, in susceptibility measurements along the $\mathrm{c}$ axis it has been reported the presence of a second maximum at temperatures lower than the SP transition temperature. [14, 44 This second maximum in the susceptibility along the $c$ axis has been attributed to the transition to a long-range $\mathrm{AF}$ order and it occurs at a temperature slightly higher than the corresponding Neél temperature $\left(\mathrm{T}_{\mathrm{N}}\right)$.

In Fig. 4 , the magnetic susceptibility computed by QMC simulations is shown as a function of temperature for a dimerized Heisenberg chain with $\delta=0.05$ in the presence of both magnetic and non-magnetic impurities. The statistical error of the QMC simulations is about the size of the open circles in this figure. The susceptibility for the pure system on a chain of 80 sites has also been computed. The results for this case are very similar to the case of $N=40$ with OBC and strong edge bonds and they are not included in the figure for clarity. $\chi(\mathrm{T})$ for $N=80$ with two spin-1 impurities has also been computed, for the case where the first impurity is located at 
site 1 and the second one at various sites such as 20,21, 40 and 41 . The susceptibilities obtained for these systems are compared with those obtained from chains of $N=40$ with open boundary conditions. The latter chain can be considered as resulting from a $N=80$ chain after a pair of nonmagnetic impurities have been introduced.

In the case of $N=40$, OBC, two possibilities have been also analyzed: the case where the bonds at the chain ends are both weak and the case where they are both strong. The results can be interpreted in terms of the intuitive picture developed in Ref. [25] for the large dimerization limit. In the case of strong end links, the spins are basically paired in strong dimers and hence it is quite likely that a spin gap is still present in the chain leading to an exponential decay of $\chi(\mathrm{T})$ as $T \rightarrow 0$ observed in the figure. This behavior is not observed in the other case of weak end bonds where there are loose spins at the ends of the chain which cause a strong suppression of the spin gap. In the case of spin-1 impurities, depending on their relative positions, a periodic chain is effectively cut, or in chains with odd number of sites, or in chains with even number of sites where at least one of them has both weak end bonds. In both cases, the susceptibility would not decay exponentially to zero as the temperature goes to zero as it is observed in the figure at least down to the lowest temperature achievable with our algorithm.

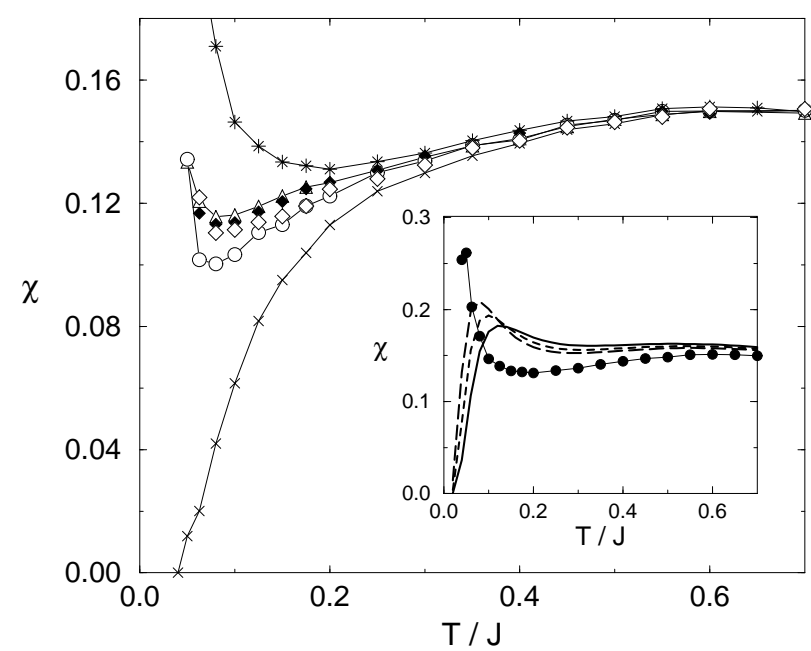

FIG. 4. Magnetic susceptibility as a function of temperature obtained by Monte Carlo simulations in dimerized Heisenberg chains with $\delta=0.05$. $N=80$, two $S=1$ impurities in sites 1 and 40 (up triangles), in sites 1 and 41 (open circles), in sites 1 and 20 (filled diamonds), and in sites 1 and 21 (open diamonds). $N=40$, OBC, weak end bonds (stars), strong end bonds (crosses). In the inset, it is shown $\chi(\mathrm{T})$ obtained by exact diagonalization in chains of $N=12$ (solid line), $N=14$ (short dashed line), and $N=16$ (long dashed line), and OBC with weak end bonds. The results obtained by QMC for $N=40, \mathrm{OBC}$, weak end bonds (circles) for temperatures down to $\mathrm{T} / \mathrm{J}=0.04$ are also shown.
In principle, the experimentally observed behavior of the susceptibility should be theoretically accounted for by averaging the results obtained for chains of even number of sites with the ones obtained for chains with odd number of sites. The latter diverge as $T \rightarrow 0$ due to the total $S^{z}=1 / 2$. For the case of nonmagnetic impurities (open boundary conditions), from both QMC and exact diagonalization results for chains up to 16 sites, we conclude that by averaging the susceptibilities of chains with even number of sites and strong end bonds with the susceptibilities of chains with odd number of sites, one does not obtain a second maximum in the low temperature region. The Curie behavior of the odd chains dominates over the exponentially decaying behavior of the even chains at low temperatures. On the other hand, the susceptibility on chains with even number of sites and weak end bonds, as it can be seen in the inset of Fig. 1, does indeed show this second maximum and it is quite likely that this second maximum can survive the averaging procedure as we have verified numerically.

Let us examine to what extent these results are relevant to spin-Peierls systems. In these systems, upon the introduction of nonmagnetic impurities, the spin-lattice coupling leads to the case of chains where the two edge bonds are strong, even for an odd total number of sites in which case a soliton is formed in the middle of the chain. 38 However, it has been recently claimed that elastic interchain interactions may stabilize a given pattern of distortions, i.e. with and without nonmagnetic impurities the weak and strong bonds of the dimerized chain may remain the same as for non-dynamical phonons 45 and this behavior has been verified numerically. [38] That is, even site chains with both weak links are still present in $\mathrm{SP}$ chains due to elastic coupling to pure neighbor chains.

Besides, it is reasonable to think that the results obtained for larger open chains are going to be indicative of smaller nonmagnetic impurity doping. Then, as it can be seen in the inset of Fig. 1 , the shift to lower temperatures of the second peak as $N$ is increased is in complete agreement with the experimental results showing that the Neél temperature decreases as the impurity concentration decreases 44]. One should notice that this behavior of $\mathrm{T}_{\mathrm{N}}$ occurs in the dimerized region of $\mathrm{CuGeO}_{3}$ where precisely our fixed-dimerization study should apply. (A different behavior in the uniform region for large impurity concentrations has been reported 44]). Finally, the fact that the second maximum in $\chi$ is due to the contribution of even site chains with weak end links is very consistent with the fact that, as mentioned above, these chains present the largest enhancement of AF correlations upon doping.

On the other hand, the susceptibility in the case of two magnetic impurities has already a very similar temperature dependence for different impurity positions and it is quite likely that an average over all these impurity positions would still result in a curve of the same shape. Based on the mapping of this problem to an effective nonmagnetic impurity chain a second maximum should 
exist in $\chi$ and it should appear at temperatures lower than the attainable ones within our QMC algorithm.

\section{CONCLUSIONS}

In this article the effects of $S=1$ magnetic impurities on dimerized AF Heisenberg chains have been analyzed, and the results have been compared with those originated by the introduction of nonmagnetic impurities. The overall effects, such as the destruction of the spin gap which reveals itself in the dynamical spin structure factor $S(q, \omega)$ (Figs. 2 and 3) and in the magnetic susceptibility (Fig. 4), are similar between the two types of impurities. However, the microscopic origin and details of the tight states formed between the impurities and the spin-1/2 sites close to it are different. This difference translates into the existence of strong AF correlations in the immediate vicinity of the $S=1$ impurity. This local behavior could eventually be experimentally detected, for example by using NMR techniques. On the other hand, the spins located at distances of two lattice spacings (or larger) from the impurity react to the spin singlet state arising from the coupling of the $\mathrm{S}=1$ impurity with its two neighboring spin-1/2 as if it were a nonmagnetic impurity. This interesting effect produces an enhancement of AF correlations in the vicinity of the $S=1$ impurities as for Zn doping, which may lead to a global stabilization of the AF state as found experimentally in $\mathrm{CuGeO}_{3}$ using several techniques.

As discussed in the previous section with respect to the case of nonmagnetic impurities, it is expected that in spin-Peierls systems the partial substitution of $\mathrm{Cu}$ ions by $\mathrm{Ni}$ ions would also change the dimerization pattern which these compounds present below the SP transition temperature. That is, the changes in the spin-spin correlations discussed in Section III would imply, due to the spin-phonon coupling, a distortion of the dimerization pattern, especially near the $\mathrm{Ni}$ impurities. Hence our results could in principle not be directly related to the experimentally observed behavior of Ni-doped $\mathrm{CuGeO}_{3}$. However, some previous studies, as well as new results which we have recently obtained, indicate that the elastic coupling between a doped chain with its neighbor undoped ones tends to stabilize the undistorted dimerized pattern and in this case our results could be directly relevant to experiments in SP systems. In this sense, the agreement of our results for the magnetic susceptibility with recent experimental results on $\mathrm{CuGeO}_{3}$ is remarkable and this leads to an interesting explanation on the origin of the second peak observed in these experiments. On the other hand, the effects of $S=1$ impurities analyzed in this paper could be also detected in the structurally dimerized compounds such as the ones indicated in the Introduction.

\section{ACKNOWLEDGMENTS}

E. D. thanks the financial support of the NSF grant DMR-9520776. J. R. wishes to thank D. Poilblanc for many useful discussions.

[1] F. D. M. Haldane, Phys. Rev. Lett. 50, 1153 (1983).

[2] J. P. Renard, et al., Europhys. Lett. 73, 3463 (1994).

[3] For a recent review see E. Dagotto and T. M. Rice, Science 271, 618 (1996). See also Physics Today, Search and Discovery, pg. 17 October 1996.

[4] M. Azuma, Z. Hiroi, M. Takano, K. Ishida, Y. Kitaoka, Phys. Rev. Lett. 73, 3463 (1994).

[5] C. K. Majumdar and D. K. Ghosh, J. Math. Phys. 10, 1388 (1969).

[6] M. Hase, I. Terasaki and K. Uchinokura, Phys. Rev. Lett. 70, 3651 (1993).

[7] J. Riera and A. Dobry, Phys. Rev. B 51, 16098 (1995).

[8] G. Castilla, S. Chakravarty and V.J. Emery, Phys. Rev. Lett. 75, 1823 (1995).

[9] See J. C. Bonner, S. A. Friedberg, H. Kobayashi, D. L. Meier, and H. W. J. Blöte, Phys. Rev. B 27, 248 (1983), and references therein.

[10] B. Lake, D. A. Tennant, R. A. Cowley, J. D. Axe, and C. K. Chen, J. Phys. Cond. Mat. 8, 8613 (1996), and references therein.

[11] Z. Hiroi, S. Amelinckx, G. Van Tendeloo, and N. Kobayashi, Phys. Rev. B 54, 15849 (1996).

[12] A. W. Garrett, S. E. Nagler, D. A. Tennant, B. C. Sales, and T. Barnes, Phys. Rev. Lett 79, 745 (1997).

[13] See for example, Y. Ajiro et al., Phys. Rev. B 51, 9399 (1995).

[14] J. G. Lussier, S. M. Coad, D. F. McMorrow, and D. McK. Paul, J. Phys. Cond. Matt. 7, L325 (1995).

[15] Y. Sasago, N. Koide, K. Uchinokura, M. C. Martin, M. Hase, K. Hirota, and G. Shirane, preprint condmat/9603185, 1996.

[16] M. Hase, I. Terasaki, Y. Sasago, K. Uchinokura, and H. Obara, Phys. Rev. Lett. 71, 4059 (1993).

[17] S. B. Oseroff, S-W. Cheong, B. Atkas, M. F. Hundley, Z. Fisk, and L. W. Rupp, Jr., Phys. Rev. Lett. 74, 1450 (1995).

[18] A. K. Hassan, L. A. Pardi, G. B. Martins, G. Cao, and L. C. Brunel, preprint cond-mat/9706286.

[19] S. Eggert and I. Affleck, Phys. Rev. B 46, 10866 (1992).

[20] S. Eggert and I. Affleck, Phys. Rev. Lett. 75, 934 (1995).

[21] G. B. Martins, M. Laukamp, J. Riera, and E. Dagotto, Phys. Rev. Lett. 78, 3563 (1997).

[22] M. Laukamp, G. B. Martins, C. J. Gazza, A. L. Malvezzi, E. Dagotto, P. M. Hansen, A. C. López, and J. Riera, Phys. Rev. B 57, 10755 (1998).

[23] A. Sandvik, E. Dagotto, and D. Scalapino, Phys. Rev. B 56, 11701 (1997).

[24] H. Fukuyama, T. Tanimoto, and M. Saito, J. Phys. Soc. Jpn. 65, 1182 (1996); H. Fukuyama, N. Nagaosa, M. 
Saito, and T. Tanimoto, J. Phys. Soc. Jpn. 65, 2377 (1996); N. Nagaosa, A. Furusaki, M. Sigrist, and H. Fukuyama, J. Phys. Soc. Jpn. 65, 3724 (1996).

[25] G. B. Martins, E. Dagotto, and J. Riera, Phys. Rev. B 54, 16032 (1996).

[26] M. Azuma, M. Takano, and R. S. Eccleston, preprint cond-mat/9706170. See also M. C. Martin, M. Hase, K. Hirota, G. Shirane, Y. Sasago, N. Koide, and K. Uchinokura, preprint.

[27] P. Lemmens, M. Fisher, G. Güntherodt, C. Gros, P. G. J. van Dongen, M. Weiden, W. Richter, C. Geibel, and F. Steglich, preprint cond-mat/9703060.

[28] M. Azuma, Y. Fujishiro, M. Takano, T. Ishida, K. Okuda, M. Nohara, and H. Takagi, Phys. Rev. B 55, R8658 (1997); M. Nohara, H. Takagi, M. Azuma, Y. Fujishiro, and M. Takano, preprint.

[29] Y. Motome, N. Katoh, N. Furukawa, and M. Imada, J. Phys. Soc. Jpn. 65, 1949 (1996); M. Sigrist and A. Furusaki, J. Phys. Soc. Jpn. 65, 2385 (1996); H.-J. Mikeska, U. Neugebauer, and U. Schollw" ock, preprint cond-mat/9608100; T. K. Ng, Phys. Rev. B 54, 11921 (1996); T. K. Ng, preprint cond-mat/9610016; Y. Iino and M. Imada, J. Phys. Soc. Jpn. 65, 3728 (1996); M. Imada and Y. Iino, J. Phys. Soc. Jpn. 65, 568 (1997); K. Hida, preprint cond-mat/9612232 T. Miyazaki, M. Troyer, M. Ogata, K. Ueda, and D. Yoshioka, preprint cond-mat/9706123; M. Fabrizio and R. Mélin, preprint cond-mat/9703102; and references therein.

[30] S. Miyashita and S. Yamamoto, Phys. Rev. B 48, 913 (1993); Erik Sorensen and Ian Affleck, Phys. Rev. B 49, 15771 (1994); and references therein.

[31] M. Fabrizio and R. Mélin, preprint cond-mat/9706158, 1997.

[32] G. E. Granroth et al., preprint cond-mat/9710161, 1997.

[33] I. Affleck et al., Phys. Rev. Lett. 59, 799 (1987).

[34] P. W. Anderson, Mater. Res. Bull. 8, 153 (1973).

[35] S.A. Kivelson, D.S. Rokhsar, and J.P. Sethna, Phys. Rev. B 35, 8865 (1987).

[36] D. Augier, D. Poilblanc, E. Sorensen, I. Affleck, preprint cond-mat/9802053.

[37] A. E. Feiguin, J. A. Riera, A. Dobry, H. A. Ceccatto, Phys. Rev. B 57, 14607 (1997).

[38] P. Hansen, D. Augier, J. Riera, and D. Poilblanc, preprint cond-mat/9805325.

[39] E. Dagotto, Rev. Mod. Phys. 66, 763 (1994).

[40] E. Dagotto, J. Riera, A. Sandvik, and A. Moreo, Phys. Rev. Lett. 76, 1731 (1996).

[41] S. Sugai et al., Phys. Rev. B 42, 1045 (1990).

[42] J. E. Hirsch et al., Phys. Rev. B 26, 5033 (1982).

[43] P. E. Anderson, J. Z. Liu, and R. N. Shelton, Phys. Rev. B 56, 11014 (1997).

[44] T. Masuda, et al., Phys. Rev. Lett. 80, 18 May (1998); K. Manabe, et al., preprint cond/mat/9805072.

[45] D. Khomskii, W. Geertsma, and M. Mostovoy, Czechoslovak Journal of Physics, vol. 46 (1996), Suppl. S6 (Proceedings of the 21st International Conference on Low Temperature Physics, Prague, August 1996). 